\title{
Streptococcus gallolyticus-induced "kissing abscess" of anterior mitral valve leaflet and intervalvular fibrosa from an aortic valve endocarditis - a case of delayed diagnosis
}

\author{
Andreea-Mihaela Ignat ${ }^{1}$, Andreea-Maria Ursaru ${ }^{1}$, Dana Corduneanu ${ }^{2}$, Irina luliana \\ Costache $^{*, 1,3}$, Marilena Spiridon ${ }^{1}$, Antoniu Octavian Petris ${ }^{1,3}$, Alexandru Titu Ciucu ${ }^{4}$, \\ Dan-Nicolae Tesloianu ${ }^{1}$
}

${ }^{1}$ Cardiology Department, University Emergency Hospital "Sf. Spiridon", Iasi, Romania, ${ }^{2}$ Internal Medicine Department, University Emergency Hospital "Sf. Spiridon", lasi, Romania, "3"Grigore T. Popa" University of Medicine and Pharmacy, lasi, Romania, ${ }^{4}$ Cardiovascular Surgery Department, Institute of Cardiovascular Disease "George I. M. Georgescu", Iasi, Romania,

\begin{abstract}
A fatal disease until Mid-Twentieth Century, nowadays infective endocarditis is curable due to advances in diagnostic techniques, especially in echocardiography, the possibility of cardiac surgery during the active infectious process and new antibiotics. We present a 62 years-old man, with no significant past medical illness, referred for persistent cough, fatigue, shortness of breath, loss of weight and paleness, with recent history of persistent fever and positive blood cultures with Streptococcus gallolyticus. Echocardiographic examination revealed aortic and mitral valve vegetations and anterior mitral valve leaflet abscess. During surgery, the presence of a "kissing abscess" on the atrial side of the anterior mitral leaflet, arising from a vegetation on the non-coronary cusp of the aortic valve, was confirmed, together with an intervalvular fibrosa abscess. Special reinforcement with an oversewn bovine pericardial patch and reconstruction of the intervalvular fibrous body was necessary during aortic and mitral valve replacement. Despite delayed diagnosis, the case had a favorable outcome, the patient being discharged presenting a good clinical state. Numerous complications, including abscesses and early infective endocarditis on prosthetic aortic valve, would have been probably avoided with prompt recognition and early treatment of endocarditis.
\end{abstract}

Keywords: delayed diagnosis, infective endocarditis, "kissing abscess", Streptococcus gallolyticus, acute kidney injury

\section{Introduction}

"The infective endocarditis increases the physician's interest in the development of an infectious process". William Bart Osler, 1893

Infective endocarditis is a heterogenous disease with fever as a constant symptom, often with a considerable delay between the

Received: July 2016; Accepted after review: August 2016; Published: September 2016.

${ }^{*}$ Corresponding author: Assoc. Prof. Irina Iuliana Costache, MD PhD, Cardiology Department, University Emergency Hospital "Sf. Spiridon", Grigore T. Popa" University of Medicine and Pharmacy, Iasi, Romania Email: irinaiulianacostache@yahoo.com onset of clinical signs and recognition of the diagnosis [1].

We present a case of aortic and mitral valve endocarditis caused by Streptococcus gallolyticus, probably with a gastrointestinal point of entry, complicated with acute heart failure, mitral valve leaflet abscess and intervalvular fibrosa abscess, that required urgent surgery. Secondary involvement of the anterior mitral leaflet may appear in patients with infective endocarditis affecting the aortic valve because of the intimate anatomic relationship between the two valves. "Kissing lesion" is observed in $10-15 \%$ of patients with infective endocarditis of the aortic valve, due to infected jet of aortic regurgitation hitting the 
ventricular surface of the leaflet [2, 3]. Infective endocarditis affecting the aortic valve may also lead to secondary involvement of the intervalvular fibrosa. The MAIVF (mitral-aortic intervalvular fibrosa) is a thin, membranous area located between the aortic root and the left atrioventricular orifice and, due to its location, it can be affected in aortic valve endocarditis [4]. A case with initial unfavorable postoperative evolution (early prosthetic valve endocarditis) due to delayed diagnosis, fortunately had a good outcome at discharge and during follow-up.

\section{Case report}

A 62 years-old man presented to the Cardiology Department with shortness of breath, productive cough, fatigue and swollen legs. His medical history started one month before the current admission, when he addressed to the Gastroenterology Department for dizziness and unexplained weight loss. The hematological parameters revealed mild normocytic normochromic anemia and a nonspecific inflammatory syndrome was present. The initial suspected diagnostic of gastric or colonic neoplasia was refuted by endoscopic investigations and low blood level of specific tumor markers. Because during this hospitalization the patient had high fever $\left(38.90{ }^{\circ} \mathrm{C}\right)$ and started to cough, blood cultures were taken, revealing Streptococcus gallolyticus, subspecies gallolyticus susceptible to penicillin (minimum inhibitory concentration $<0,125 \mathrm{mg} / \mathrm{L})$. Chest X-ray showed bilateral reticulonodular infiltrates. Chest-abdomen-pelvis CT scan ruled out a small bowel cancer, but raised two others diagnostic hypotheses, pulmonary tuberculosis and bronchopneumonia (Figure 1); thereby the patient was transferred to the Pneumology Department, where paraclinical investigations excluded pulmonary tuberculosis. Anemia, however, worsened, requiring blood transfusion, which was complicated with acute pulmonary edema. There was a reconsideration of the diagnosis, infective endocarditis being the main supposition, which brought the patient to the Cardiology Department.

On current clinical presentation the patient was apyretic, with pale skin and mucous membranes, swollen legs and profuse diaphoresis. During physical examination crackles were heard from the lung bases to the midline of both left and right lungs, the patient having tachypnea and abdominal breathing. A Levine grade 3 pansystolic murmur was best heard at the apex, and an early high-frequency diastolic murmur was also heard along the left lower sternal border and in the second right intercostal space. It was the first time when abnormal heart murmurs were detected. The patient was tachycardic, with low diastolic blood pressure (20 $\mathrm{mmHg}$ ) and normal systolic component $(120 \mathrm{mmHg})$, prominent peripheral pulses and tender hepatomegaly. There were no peripheral secondary signs of endocarditis like splinter hemorrhages, Osler nodes or Janeway lesions. Biochemical parameters revealed high uric acid blood level, high blood urea nitrogen and serum creatinine levels, along with important biological inflammatory syndrome. Electrocardiographic recording showed sinus tachycardia (HR = 100 beats $/ \mathrm{min}$ ) and a first degree atrioventricular block.

Because there was a high suspicion of infective endocarditis, a two-dimensional transthoracic echocardiography (2D TTE) was performed, which revealed a mobile hyperechoic mass $(>10 \mathrm{~mm})$ attached to the aortic leaflets (Figure 2) and thickened anterior mitral leaflet (on its septal insertion). Severe aortic and mitral regurgitation were found on Doppler examination, with preserved left ventricular ejection fraction.

Transesophageal echocardiography (TEE) confirmed the presence of vegetation on the non-coronary cusp of the aortic valve, measuring $14 \mathrm{~mm}$ in diameter (Figure 3). An abscess cavity on the atrial side of the anterior mitral valve leaflet, where the mobile vegetation on the non-coronary cusp of the aortic valve was in contact with the mitral valve (anterior mitral valve abscess) (Figure 4) was also found. 


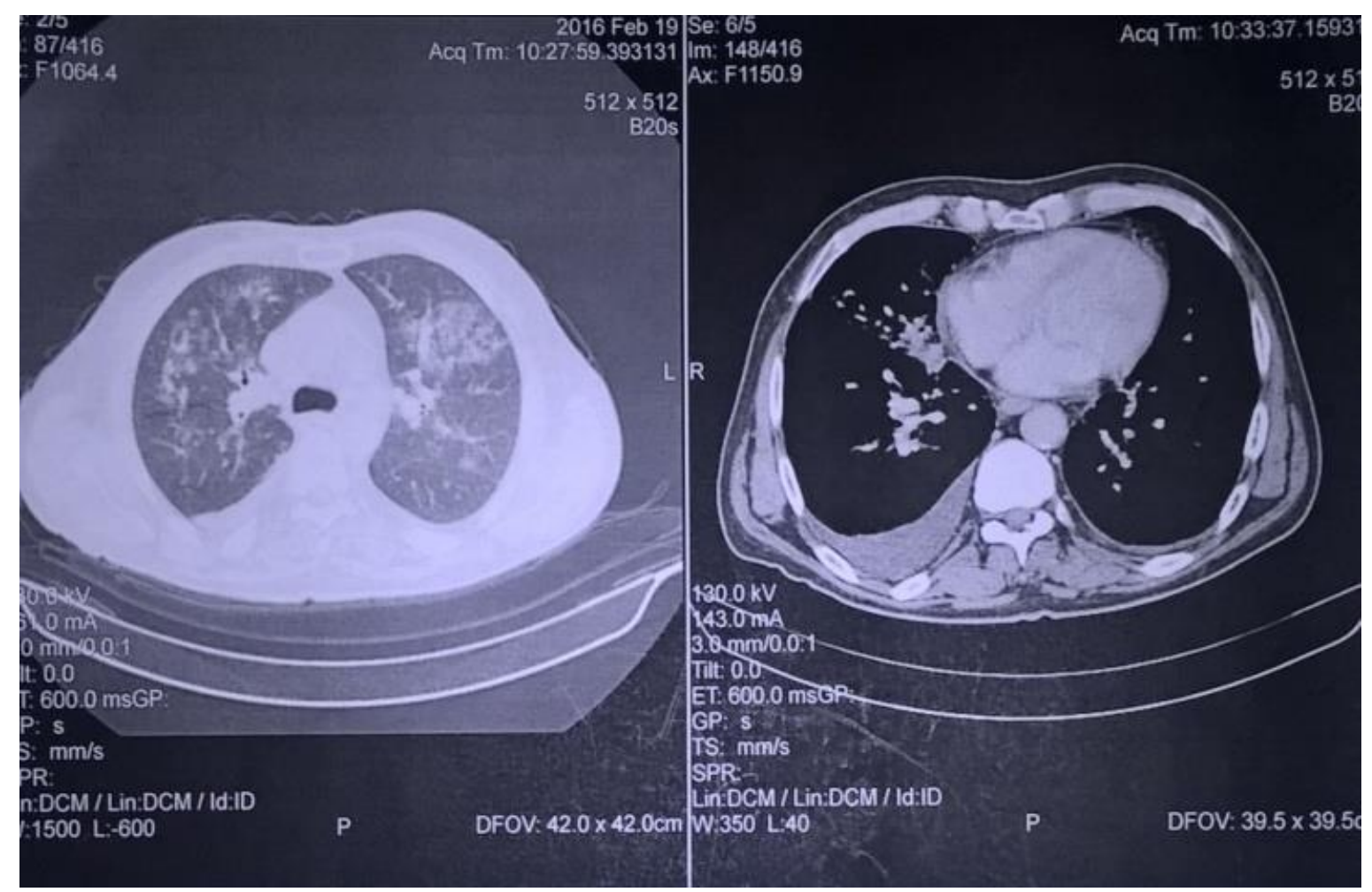

Fig. 1. Thoracic CT scan native phase (left) and with contrast (right): reticulonodular infiltrates in both lungs.

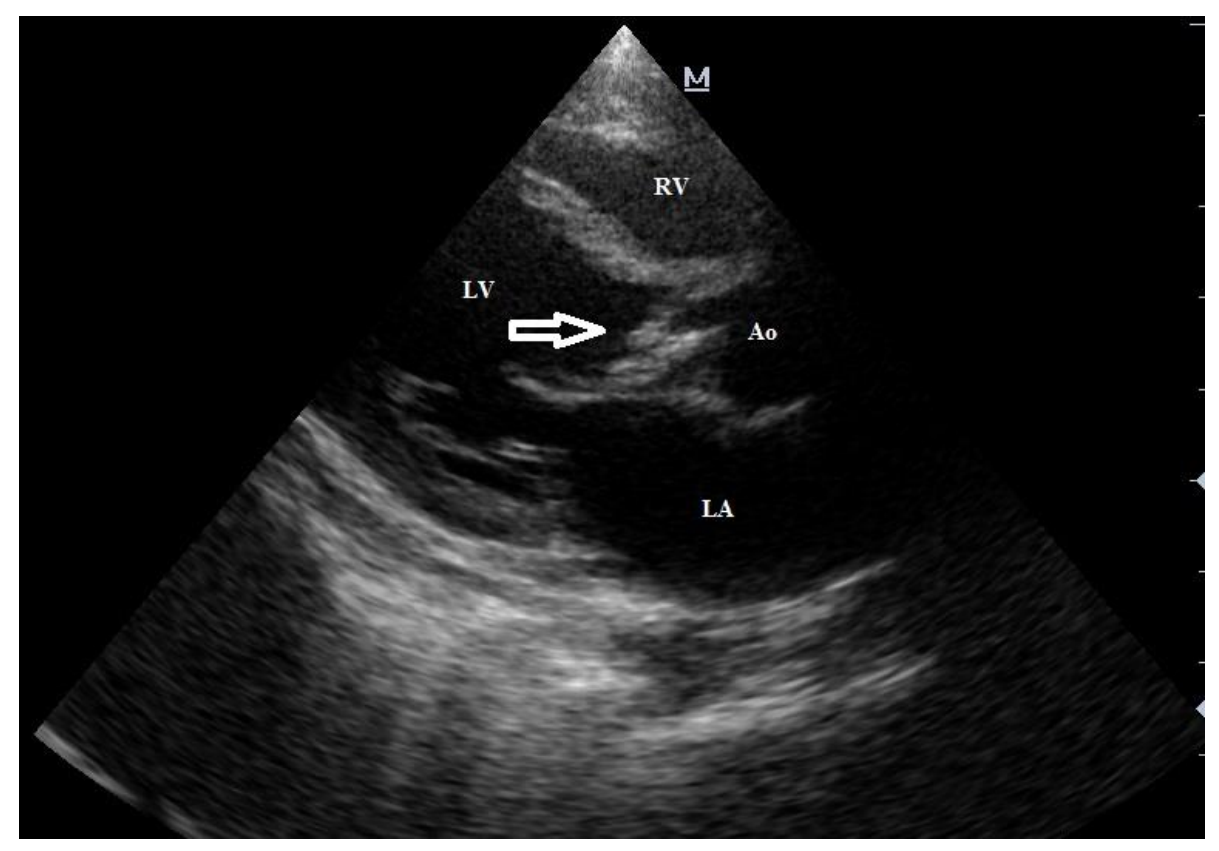

Fig. 2. TTE-Parasternal long axis section showing hyperechoic mass attached to the aortic leaflets (arrow), $L A=l$ eft atrium, $L V=$ left ventricle, $A o=$ aorta, $R V=$ right ventricle. 


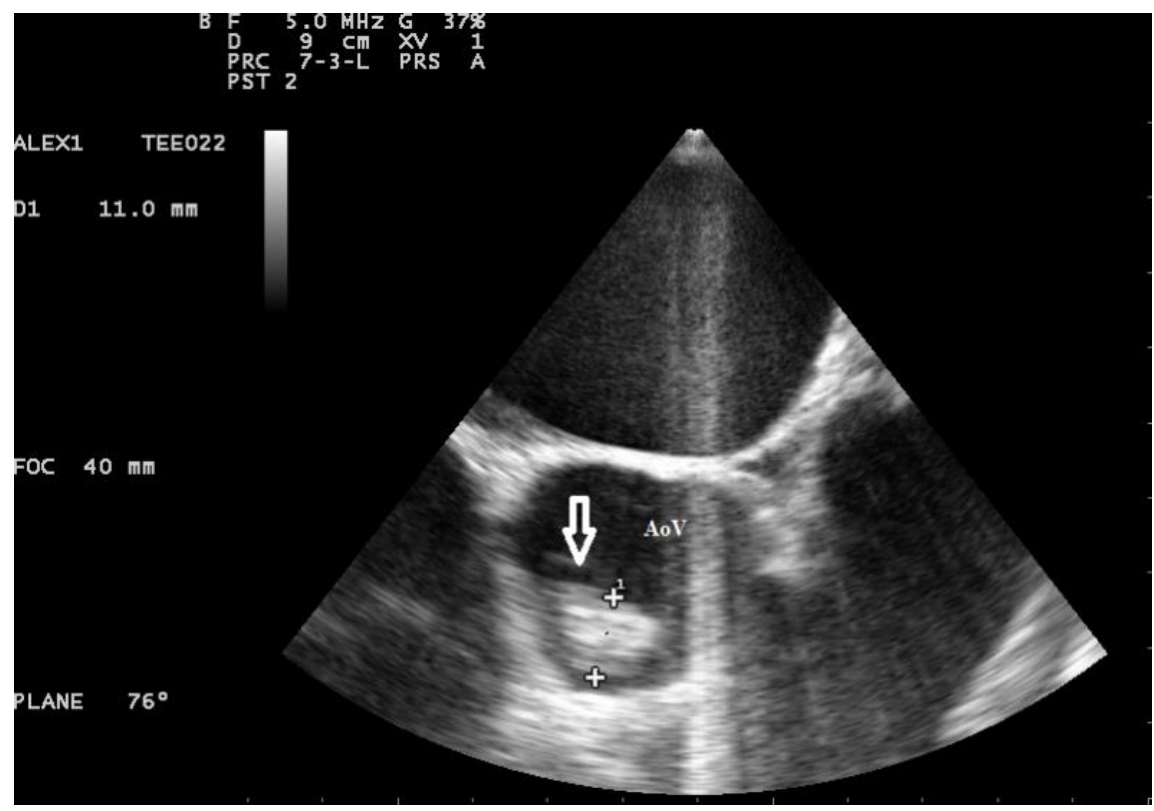

Fig. 3. TEE section: Vegetation on the non-coronary cusp of the aortic valve (arrow).

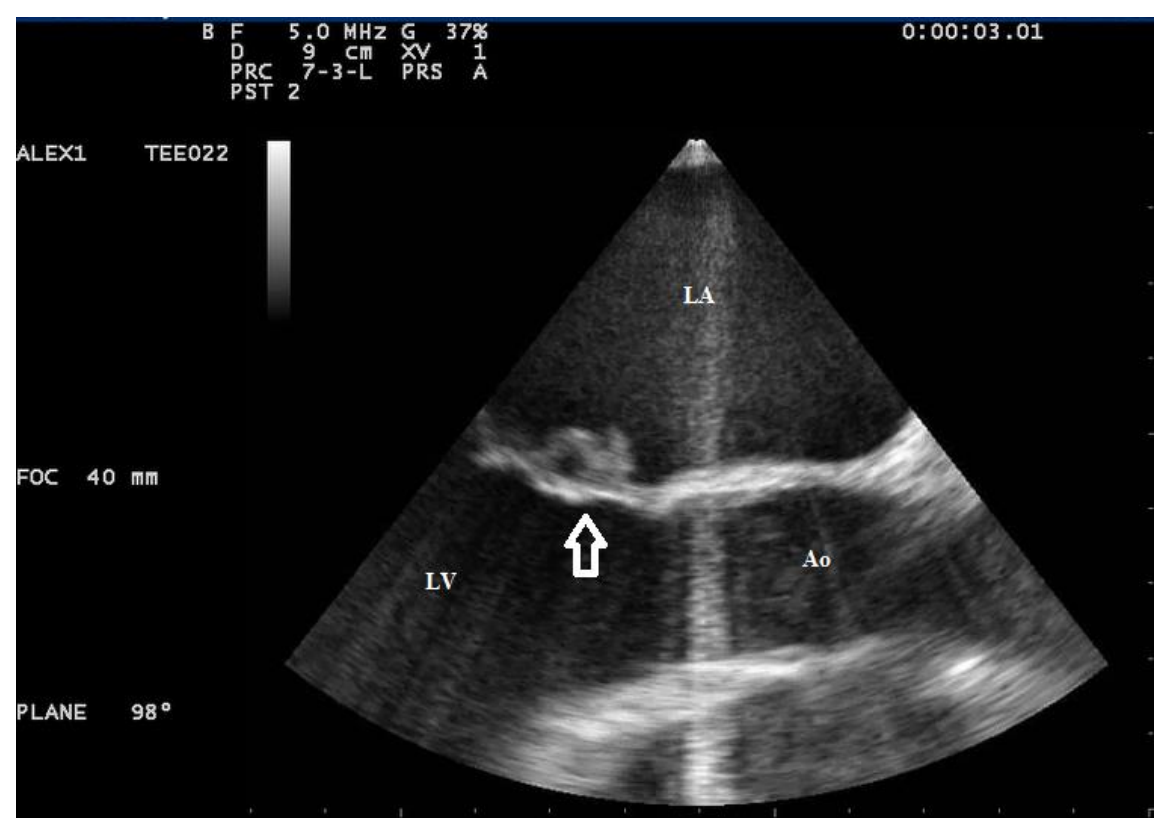

Fig. 4. TEE section: abscess on the atrial side of the anterior mitral leaflet (arrow).

We initiated antibiotherapy with penicillin G (20 million units/day) and gentamicin (240 $\mathrm{mg} /$ day), knowing the etiological agent of endocarditis (Streptococcus gallolyticus, subspecies gallolyticus). The presence of congestive heart failure due to valvular regurgitations, uncontrolled infection with mitral valve leaflet abscess and the large mobile vegetations localized on the aortic valve, were all indications for urgent surgery.
After aortic vegetation and MAIVF excision (intraoperative diagnosis of intervalvular fibrosa abscess), double replacement of aortic and mitral valve was performed with bileaflet mechanical prosthesis. In the seventh day postoperatively, the blood cultures turned negative, but with increased blood urea nitrogen levels, increased serum creatinine and hepatic cytolysis syndrome, which persisted for two weeks, then turned to normal. Moderate systolic dysfunction and 
mild to moderate diastolic dysfunction were noticed immediately after surgery, but the left ventricular ejection fraction improved during next month, returning to normal. Peripheral embolism occurred probably during surgery, with cyanosis of the third finger of the left hand initially, evolving later with dry gangrene, treated by necrectomy.

Two weeks after surgery the patient was redirected to the Cardiology Department to continue his treatment under medical supervision. His clinical status oscillated, one of the main problems being severe anemia (serum $\mathrm{Hb}=6.3 \mathrm{~g} / \mathrm{dl}$ ), which required blood transfusions with 3 units of blood in three consecutive weeks, one each week. The source of bleeding was found to be bleeding from gastric and colonic angiodysplasia, revealed by endoscopy. Echocardiographic monitoring performed weekly during follow-up, raised the supposition on the 18th day from admission (under therapy with penicillin G - 20 million units/day) of early infective endocarditis on prosthetic aortic valve. Paraprosthetic aortic leak (Figure 5) due to partial aortic prosthesis dehiscence and severe aortic regurgitation were detected (Figures 5 and 6).

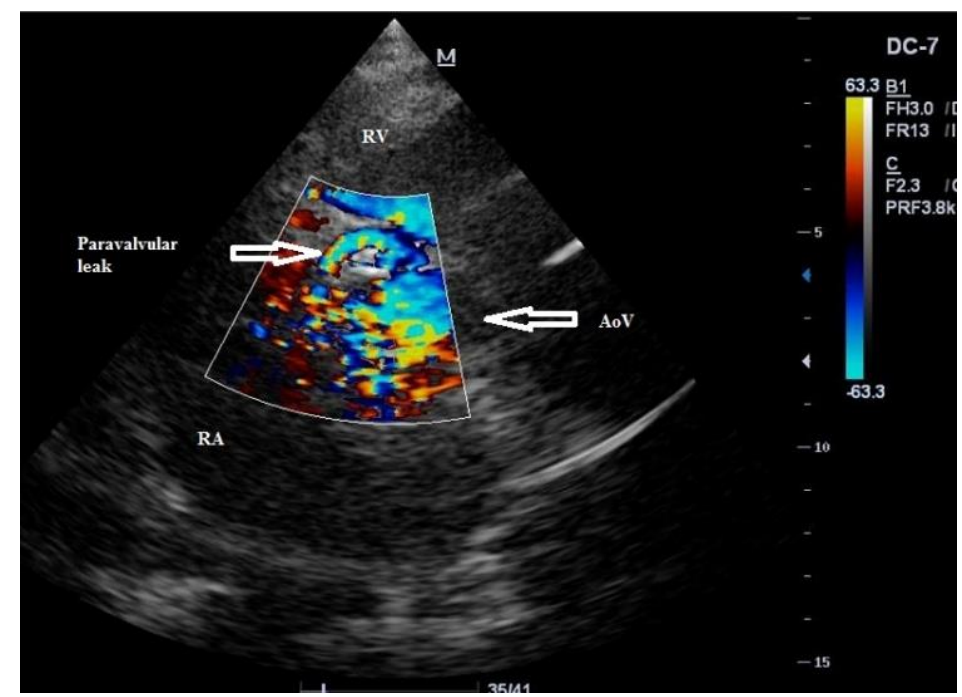

Fig. 5. Doppler echocardiography at the aortic valve level showing paravalvular leak of the aortic mechanical prosthesis.

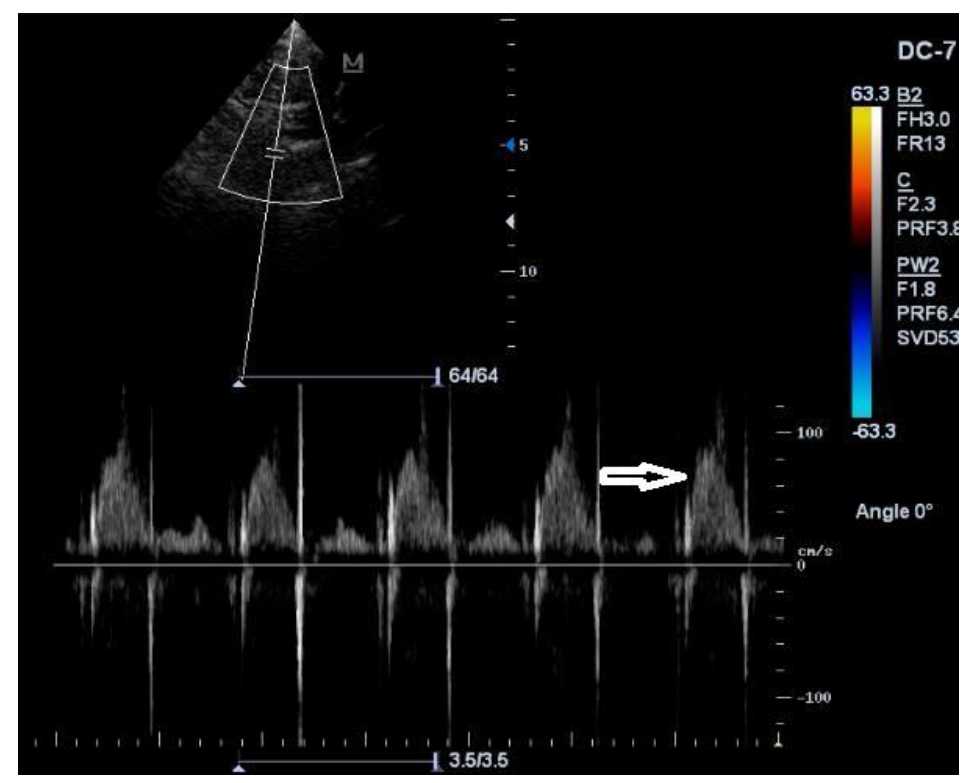

Fig. 6. Pulsed wave (PW) Doppler - Retrograde flow in the descending aorta - holodiastolic flow, $\mathrm{V} \max >80 \mathrm{~cm} / \mathrm{s}$ 
Blood cultures with methicillin-resistant Staphylococcus aureus turned positive. Instead of an expected deterioration, we observed an enhanced left ventricular contractility.

The patient was hemodynamically stable, without clinical signs or symptoms of heart failure. The cardiovascular surgeon temporized a reintervention for aortic prosthesis replacement, at least for the next 6 months, because he estimated a high patientassociated risk, so that the risk would have outweighed the benefits. Antibiotic therapy with Rifampin (1200 mg/day), Gentamicin (240 $\mathrm{mg} /$ day) and Vancomycin (2 g/day) was initiated, but after two weeks the patient developed acute kidney injury (serum creatinine $=3.2 \mathrm{mg} / \mathrm{dl}$, creatinine clearance $=$ $29.3 \mathrm{ml} / \mathrm{min}$ ), therefore being transferred to the Nephrology Department. There, after another three weeks of hospitalization and proper treatment, blood urea nitrogen level and serum creatinine significantly decreased. The clinical evolution was favorable without the need for surgical reintervention, despite all negative prognostic factors. Stable clinical condition (moderate dyspnea on exertion, no clinical signs of heart failure), negative blood cultures (after 1 week), preserved left ventricular ejection fraction and cavity dimensions at echocardiographic assessment, were noticed, at one and three months post-discharge follow up. Severe aortic regurgitation remains the patient's major problem.

\section{Discussions}

This case report describes the evolution of an appropriately treated patient with native valve endocarditis, with a positive outcome in the end, but with multiple complications due to delayed diagnosis. What we are trying to highlight, is that time to diagnosis is crucial for the prognosis, and therefore, it is very important to refer the patients early to echocardiography. In reality this is not always done, because symptoms can be difficult to interpret or recognize (in this particular case, abnormal heart murmurs were first identified only three weeks after the first medical consultation, at the moment of admission in the Cardiology Department).

In $23 \%$ of cases of infective endocarditis, the diagnosis is made after more than 30 days from the onset of symptoms [1]. Even in hospitalized patients, there is often a delay before echocardiography is being performed, because of the atypical presentation symptoms. The medium delay is of 8.2 days from admission [5]. For our patient, unfortunately the delay was 23 days.

We would like to underline the importance and advantages of echocardiography in endocarditis.

Other imaging modalities such as MRI or CT-scan can also, or at least sometime, visualize the vegetation. The patient had a CT scan and no vegetation or heart problem were noticed. This indicates the low sensitivity of other imagistic methods, other than echocardiography.

A patient with undifferentiated febrile syndrome and bacteremia is characterized as being at initially low risk of likelihood for infective endocarditis. But this likelihood would have been dramatically increased by the appearance of a significant new heart murmur $[6,7]$. Unfortunately, in our patient, the heart murmurs were detected after a 3-weeks delay. While investigations have become more elaborate, in every day practice physical exams have often become more superficial [5]. Maybe because we tend to underestimate the importance of clinical examination in favor of paraclinical investigations, we lose important clues to diagnosis. Laboratory investigations must be headed by complete, extensive and thorough physical exam.

A particularity of the case is the formation of a "kissing abscess" of the anterior mitral leaflet, occurring by extension from an infected regurgitation aortic jet striking the ventricular surface of the anterior mitral valve leaflet or by direct contact between the mitral leaflet and the aortic vegetation. These mechanisms lead to abscesses localized on the ventricular side of the anterior mitral valve leaflet. When the lesion is on the atrial side of the leaflet, the spread of infection is not via aortic vegetation touch, but it is related to the anatomic contiguity of the aortic and mitral valves $[3,8$, 9]. For this case, Gülşen et al [3] used the 
term "reverse kissing vegetation", the same aspect being revealed in our patient by TEE. Several other cases of "kissing abscess" of anterior mitral valve and MAIVF have been reported. Easaw et al [10] have presented a similar case, but with an uneventful recovery after aortic and mitral valve replacement. In other cases, the mitral valve abscess has led to aneurysm of the anterior leaflet, complicated with perforation of the valve and severe mitral regurgitation, as described by Fazlinezhad et al [2].

Another particularity of the case is related to the etiology of the endocarditis, first $S$. gallolyticus native valve endocarditis and $S$. aureus prosthetic valve endocarditis, afterwards. S. gallolyticus, subspecies gallolyticus is found in the gastrointestinal tract of birds, ruminants, and in a small proportion $(2.5 \%-15 \%)$ in humans, with the infection remaining subclinical in most individuals due to low virulence [11- 14]. However, in a very small fraction of patients, it benefits from adenomatous epithelial tissue to translocate through the epithelium via a paracellular mechanism, so these infections can become clinically manifested through the effective formation of resistant bacterial vegetations, which present as endocarditis [15]. It is demonstrated that $S$. gallolyticus endocarditis is related to colon cancer, so in every case a colonoscopic screening is indicated $[8,16]$. In this case, the patient had a negative diagnostic colonoscopy.

S. aureus causes up to $90 \%$ of staphylococcal infective endocarditis and is the most common cause of acute infective endocarditis, becoming the leading cause of infective endocarditis worldwide. Rates of $S$. aureus infections, particularly bacteremia associated with health care contact have increased in hospitalized patients [8]. Only a fraction of these patients will develop endocarditis. Populations at increased risk include patients with type 1 diabetes, patients on dialysis and patients with recent surgical incisions, including median sternotomy for valve replacement. $S$. aureus is a highly virulent organism and infective endocarditis with $S$. aureus is frequently fulminant and results in major local complications such as valve destruction and perivalvular extension, leading to partial or total prosthesis dehiscence when prosthetic valves are present [7].

Staphylococcus aureus, both methicillinsusceptible $S$. aureus and methicillin-resistant $S$. aureus (MRSA) strains, are the most frequent pathogens causing prosthetic valve endocarditis.

Prior history of endocarditis is an important risk factor for prosthetic valve endocarditis, which has an unfavorable prognosis and an inhospital mortality of $46 \%$ for the antibiotic treated group and $24 \%$ for the surgically treated group [17, 18]. This percentage is even higher with abscess formation history, which increases the risk of systemic embolism (systemic embolism raises the mortality up to $80 \%$ ) [1]. Both complications were present in our patient (abscess of MAIVF and left middle finger embolus).

\section{Conclusions}

From this case we conclude that transthoracic echocardiography should be performed to every patient with persistent fever of unknown origin and significant new heart murmur, to exclude a cardiac etiology, and especially infective endocarditis. Although antibiotics represent the main treatment resource in infective endocarditis, in the stage of complications like severe valvular regurgitations, presence of massive vegetations or an abscess formation, surgical intervention is the only option to obtain a favorable long-term prognosis. Despite appropriate medical care, the prognosis is dramatically reduced by delayed diagnosis, this kind of patients having a rather poor outcome. Despite the multitude of complications this patient had, antibioticsinduced acute kidney injury, peripheral embolism, mitral and intervalvular fibrosa abscesses, acute pulmonary edema from transfusions, gastrointestinal bleeding, prosthesis endocarditis and secondary aortic prosthesis dehiscence, he somehow managed to survive.

\section{Conflict of interest}

Authors declare no conflicts of interest. 


\section{References}

1. Murdoch DR, Corey GR, Hoen B, et al. Clinical presentation, etiology, and outcome of infective endocarditis in the $21^{\text {st }}$ century: the International Collaboration on Endocarditis-Prospective Cohort Study. Arch Intern Med 2009; 169:463-473.

2. Fazlinezhad A, Mirzaeei A, Alvandi Azari M. Ruptured mitral valve kissing abscess to left atrium in course of aortic valve endocarditis. Shiraz E Med J 2011; 30:112-117.

3. Gülşen K, Duygu H, Cerit L. A new entity in an immunosuppressive patient: Giant reverse kissing vegetation. Int J Cardiol 2015; 201 :279_ 281.

4. Fazlinezhad A, Fatehi $\mathrm{H}$, Tabaee $\mathrm{S}$, et al. Pseudoaneurysm of mitro-aortic intervalvular fibrosa during the course of mitral valve endocarditis with aorto-left ventricle outflow tract fistula. J Saudi Heart Assoc 2012; 24(3):201-204.

5. Cecchi E, Forno D, Imazio M, et al. New trends in the epidemiological and clinical features of infective endocarditis: results of a multicenter prospective study. Ital Heart J 2004; 5(4):24956.

6. Flegel $\mathrm{KM}$. Does the physical examination have a future? Can Med Assoc J 1999; 161 (9):1117-1118.

7. Larry MB, William KF, Rakesh MS, et al. Braunwald's Heart Disease a textbook of cardiovascular disease. $10^{\text {th }}$ ed. Philadelphia: Elsevier Saunders; 2015: 1524-1543.

8. Laurence HC, Piper C, Hetzer R, et al. Textbook of cardiac surgery in adults. $3^{\text {rd }}$ Edition. Boston: McGraw-Hill; 2008.

9. Lee CC, Siegel RJ. Sinus of valsalva pseudoaneurysm as a sequela to infective endocarditis. Tex Heart Inst $J$ 2016; 43(1):4648.
10. Easaw J, El-Omar M, Ramsey M. "Kissing abscess" of the anterior mitral valve leaflet from a vegetation on the non-coronary aortic cusp. Heart 2001; 86(1): 90.

11. Corredoira J, Alonso MP, Coira A, et al. Characteristics of Streptococcus bovis endocarditis and its differences with Streptococcus viridans endocarditis. Eur J Clin Microbiol Infect Dis 2008; 27:285-291.

12. Abdulamir AS, Hafidh RR, Abu Bakar F. The association of Streptococcus bovis/gallolyticus with colorectal tumors: the nature and the underlying mechanisms of its etiological role. $J$ Exp Clin Cancer Res 2011; 30:11.

13. Klein RS, Recco RA, Catalano MT, et al. Association of Streptococcus bovis with carcinoma of the colon. N Engl J Med 1977; 297:800-2.

14. Abeni C, Rota L, Ogliosi C, et al. Correlation among Streptococcus bovis, endocarditis and septicemia in a patient with advanced colon cancer: a case report. J Med Case Rep 2013; 7:185.

15. Boleij A, Muytjens CMJ, Bukhari SI, et al. Novel clues on the specific association of Streptococcus gallolyticus subsp gallolyticus with colorectal cancer. J Infect Dis 2011; 203(8):1101-1109.

16. Ferrari A, Botrugno I, Bombelli $E$, et al. Colonoscopy is mandatory after Streptococcus bovis endocarditis: a lesson still not learned. World J Surg Oncol 2008; 6:49.

17. Chu VH, Cabell $\mathrm{CH}$, Benjamin DK Jr, et al. Early predictors of in-hospital death in infective endocarditis. Circulation 2004; 109(14):1745-9.

18. Akowuah EF, Davies W, Oliver $S$, et al. Prosthetic valve endocarditis: early and late outcome following medical or surgical treatment. Heart 2003; 89(3):269-272. 